IFUM $657 / \mathrm{FT}$

CAMS/00-04

hep-th/0004077

\title{
Entropy of Black Holes in $D=5, N=2$ Supergravity and AdS Central Charges
}

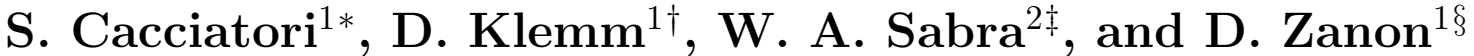

\author{
${ }^{1}$ Dipartimento di Fisica dell'Università di Milano and \\ INFN, Sezione di Milano, Via Celoria 16, 20133 Milano, Italy. \\ ${ }^{2}$ Center for Advanced Mathematical Sciences (CAMS) and \\ Physics Department, American University of Beirut, Lebanon.
}

\begin{abstract}
We consider general black holes in $D=5, N=2$ supergravity coupled to vector multiplets, and discuss the issue of microstate counting from various viewpoints. The statistical entropy is computed for the near-extremal case using the central charge of the $A d S_{2}$ factor appearing in the near-horizon geometry. Furthermore, we explicitly construct the duality transformation connecting electrically charged black holes to magnetically charged black strings, under which the $A d S_{2} \times S^{3}$ near horizon geometry becomes $A d S_{3} \times S^{2}$. For $A d S_{3}$ the counting of microstates correctly reproduces the Bekenstein-Hawking entropy, thus resolving the discrepancy previously found for $A d S_{2}$.
\end{abstract}

*email: cacciatori@mi.infn.it

†email: dietmar.klemm@mi.infn.it

‡email: ws00@aub.edu.lb

$\S$ email: daniela.zanon@mi.infn.it 


\section{Introduction}

The study of black hole solutions in $N=2$ five-dimensional supergravity coupled to vector and hypermultiplets plays an important role in the understanding of the non-perturbative structure of string and M-theory [1, 2]. In this setting the interplay between classical and quantum results is exemplified at its best.

In this paper we consider general charged black holes of the $D=5, N=2$ theories, not necessarily those obtained from compactification of eleven-dimensional supergravity on a Calabi-Yau threefold. The analysis is simplified by the rich geometric structure of the $N=2$ theories. Black hole solutions are given in terms of a rescaled cubic homogeneous prepotential which defines very special geometry [3]. In the extremal BPS case, half of the vacuum supersymmetries are preserved, while at the horizon supersymmetry is fully restored [4].

In five dimensions the supergravity action contains a Chern-Simon term which allows the existence of black holes with nonvanishing angular momenta (but still nonrotating horizon) [5, 6, 7, 8].

These issues have been the object of recent, extensive studies. In the present paper we focus on the asymptotic symmetries of the near-horizon geometry of the general near-extremal solution: the aim is the computation of the entropy from a counting of microstates to be compared to the macroscopic, thermodynamical entropy.

We will see that the calculation of the microscopic entropy of small excitations above extremality is equivalent to a microstate counting for certain black holes in two-dimensional anti-de Sitter space. The latter, however, is problematic: $A d S_{2}$ has two timelike boundaries, but when applying Cardy's formula for the density of states only one boundary is taken into account. This procedure leads to a statistical entropy result which is off by a $\sqrt{2}$ factor with respect to the Bekenstein-Hawking entropy [9]. Up to now, no satisfactory explanation of this mismatch is known. Our results support the point of view that only the ground state has an effective description in terms of a quantum-mechanical system [10], whereas the excitations above extremality are described by a two-dimensional conformal field theory [11.

In our work we address these issues in a constructive approach. The main result that we present is an explicit duality transformation, which realizes an invariance of the $N=2$ supergravity action. This duality turns the $A d S_{2} \times S^{3}$ near horizon geometry of the extremal black hole solution into $A d S_{3} \times S^{2}$. The key point underlying the duality is the fact that the three-sphere can be written as a Hopf fibration over the base $S^{2}$. For $A d S_{3}$, the counting of microstates is performed using Cardy's formula and it is shown that it reproduces correctly the Bekenstein-Hawking entropy, thus resolving the discrepancy previously found for $A d S_{2}$.

In the case where the $D=5, N=2$ supergravity action is obtained by Calabi-Yau (CY) compactification of M-theory, the considered duality transformation, which maps electrically charged black holes onto magnetically charged black strings, corresponds to the duality between M2 branes wrapping CY two-cycles and M5 branes wrapping CY four-cycles. According to [12], M-theory compactified on $A d S_{3} \times S^{2} \times M$, where $M$ 
denotes some Calabi-Yau threefold, is dual to a $(0,4)$ superconformal field theory living on an M5 brane wrapping some holomorphic CY four-cycle. This fact has been used in [13 to compute the entropy of five-dimensional BPS black holes币. We stress that our method for microstate counting applies to any near-extremal black hole in $N=2, D=5$ supergravity, independent of whether it is obtained by CY compactification or not.

Our paper is organized as follows: in section 2, the basic notions of $N=2, D=5$ supergravity and very special geometry relevant to our analysis are summarized. In section 3 we review the black hole solutions and consider the $S T U$ model as a simple example, which nonetheless retains all the interesting features of the general solutions. In section 4 we present the isometry superalgebra which arises in the near horizon limit, while in section 5 we show that the motion of a particle which moves near the horizon of the extremal rotating black hole is described by conformal quantum mechanics. This indicates that the ground state may have a description in terms of conformal quantum mechanics [15, 16], even when rotation is included. In section 6 we compute the statistical entropy of small excitations near extremality, using the $A d S_{2}$ central charge [9], and find a $\sqrt{2}$ factor of discrepancy as compared to the thermodynamical Bekenstein-Hawking result. In section 7 we construct the duality transformation for the supergravity action, and in the following section we finally perform the state counting, using the fact that the nearhorizon geometry of the dual solution includes an $A d S_{3}$ factor. In this way, we obtain a microscopic entropy which agrees precisely with the corresponding thermodynamical result. We conclude with some final remarks.

\section{$2 \quad D=5, N=2$ Supergravity and Very Special Geom- etry}

The theory of $N=2$ supergravity theory coupled to an arbitrary number $n$ of Maxwell supermultiplets was first considered in [17]. In the analysis of [17], it was established that the scalar fields of the vector multiplets parametrize a Riemannian space. The homogeneous symmetric spaces take the form

$$
\mathcal{M}=\frac{\operatorname{Str}_{0}(J)}{\operatorname{Aut}(J)}
$$

where $\operatorname{Str}_{0}(J)$ is the reduced structure group of a formally real unital Jordan Algebra of degree three, Aut $(J)$ is its automorphism group.

The scalar manifold can be regarded as a hypersurface, with vanishing second fundamental form of an $(n+1)$-dimensional Riemannian space $\mathcal{G}$ whose coordinates $X$ are in correspondence with the vector multiplets including that of the graviphoton. The equation of the hypersurface is $\mathcal{V}=1$ where $\mathcal{V}$, the prepotential, is a homogeneous cubic polynomial in the coordinates of $\mathcal{G}$,

\footnotetext{
${ }^{1}$ The work in $[13$ includes as a special case also the results obtained in 14.
} 


$$
\mathcal{V}(X)=\frac{1}{6} C_{I J K} X^{I} X^{J} X^{K}
$$

Non-simple Jordan algebras of degree three are of the form $\mathbb{R} \oplus \Sigma_{n}$, where $\Sigma_{n}$ is the Jordan algebra associated with a quadratic form. The corresponding symmetric scalar manifolds are

$$
\mathcal{M}=S O(1,1) \times \frac{S O(n-1,1)}{S O(n-1)}
$$

In this case, $\mathcal{V}(X)$ is factorizable into a linear times a quadratic form in $(n-1)$ scalars, which for the positivity of the kinetic terms in the Lagrangian, must have a Minkowski metric. For simple Jordan algebras, one obtains four sporadic locally symmetric spaces related to the four simple unital formally real Jordan algebras over the four division algebras $\mathbb{R}, \mathbb{C}, \mathbb{H}, \mathbb{O}$. For more details we refer the reader to 17 .

For M-theory compactification on a Calabi-Yau threefold with Hodge numbers $h_{(1,1)}$ and $h_{(2,1)}$, the five dimensional theory contains the gravity multiplet, $h_{(1,1)}-1$ vector multiplets and $h_{(2,1)}+1$ hypermultiplets. The $\left(h_{(1,1)}-1\right)$-dimensional space of scalar components of the abelian vector supermultiplets coupled to supergravity can be regarded as a hypersurface of a $h_{(1,1)}$-dimensional manifold whose coordinates $X^{I}(\phi)$ are in correspondence with the vector bosons (including the graviphoton). The defining equation of the hypersurface is as in (2.1)

$$
\mathcal{V}(X)=\frac{1}{6} C_{I J K} X^{I} X^{J} X^{K}=X^{I} X_{I}=1, \quad I, J, K=1, \ldots, h_{(1,1)} .
$$

Here $C_{I J K}$ are the topological intersection numbers of the Calabi-Yau, $X_{I}$ are the so called "dual" special coordinates.

The bosonic part of the ungauged supersymmetric $N=2$ Lagrangian which describes the coupling of vector multiplets to supergravity is given by

$$
e^{-1} \mathcal{L}=\frac{1}{2} R-\frac{1}{4} G_{I J} F_{\mu \nu}^{I} F^{\mu \nu J}-\frac{1}{2} \mathcal{G}_{i j} \partial_{\mu} \phi^{i} \partial^{\mu} \phi^{j}+\frac{e^{-1}}{48} \epsilon^{\mu \nu \rho \sigma \lambda} C_{I J K} F_{\mu \nu}^{I} F_{\rho \sigma}^{J} A_{\lambda}^{K} .
$$

The corresponding vector and scalar metric are completely encoded in the function $\mathcal{V}(X)$

$$
\begin{aligned}
G_{I J} & =-\left.\frac{1}{2} \partial_{I} \partial_{J} \ln \mathcal{V}(X)\right|_{\mathcal{V}=1} \\
\mathcal{G}_{i j} & =\left.G_{I J} \partial_{i} X^{I} \partial_{j} X^{J}\right|_{\mathcal{V}=1}
\end{aligned}
$$

where $\partial_{i}$ and $\partial_{I}$ refer, respectively, to partial derivatives with respect to the scalar fields $\phi^{i}$ and $X^{I}=X^{I}\left(\phi^{i}\right)$. 
Further useful relations are

$$
\partial_{i} X_{I}=-\frac{2}{3} G_{I J} \partial_{i} X^{J} \quad, \quad X_{I}=\frac{2}{3} G_{I J} X^{J} .
$$

It is worth pointing out that for Calabi-Yau compactifications, $\mathcal{V}$ represents the intersection form, $X^{I}$ and $X_{I}=\frac{1}{6} C_{I J K} X^{J} X^{K}$ correspond, respectively, to the size of the twoand four-cycles of the Calabi-Yau threefold.

\section{Black Holes in the $S T U=1$ Model}

In the last few years considerable progress has been made in the study of BPS black hole states of the low-energy effective actions of compactified string and $M$-theory. This was mainly motivated by the important role that these states play in the understanding of the non-perturbative structure of string theory. The magnetic and electric BPS solutions of five-dimensional $N=2$ supergravity models coupled to vector and hypermultiplets can be regarded as solitons interpolating between two vacua: Minkowski flat space at infinity and $A d S_{3} \times S^{2}$ and $A d S_{2} \times S^{3}$ near the horizon. At a generic point in space-time, the BPS solution breaks half of supersymmetry. However, near the horizon supersymmetry is enhanced and fully restored.

In M-theory compactified on a Calabi-Yau threefold, electrically charged point-like and magnetically charged string-like BPS states correspond to the two and five-branes of M-theory wrapped around the two- and four-cycles of the Calabi-Yau space respectively. Though the details of the low-energy Lagrangian depend very much on the geometric and topological data of the compactified Calabi-Yau space, the analysis of the BPS solutions is considerably simplified by the rich geometric structure based on "very special geometry" underlying the $N=2$ five-dimensional theories with vector supermultiplets [18].

The metric for the BPS black hole solutions can be brought to the form [8]

$$
\begin{aligned}
d s^{2} & =-e^{-4 V}\left(d t+w_{m} d x^{m}\right)^{2}+e^{2 V} d \vec{x}^{2}, \\
F_{m n}^{I} & =\partial_{m}\left(X^{I} \mathcal{Q}_{n}\right)-\partial_{n}\left(X^{I} \mathcal{Q}_{m}\right), \\
F_{t m}^{I} & =-\partial_{m}\left(e^{-2 V} X^{I}\right), \\
e^{2 V} X_{I} & =\frac{1}{3} H_{I},
\end{aligned}
$$

where $H_{I}$ are harmonic functions, $H_{I}=h_{I}+\frac{Q_{I}}{r^{2}}, h_{I}$ are constants and $Q_{I}$ denote the electric charges. Furthermore one has $\mathcal{Q}_{n}=e^{-2 V} w_{n}$, and the field strength of $w_{m}$ is self-dual. If one defines the rescaled coordinates $Y^{I}=e^{V} X^{I}$, then the underlying very special geometry implies that

$$
e^{3 V}=\frac{1}{6} C_{I J K} Y^{I} Y^{J} Y^{K} .
$$

As a general magnetic string solution of $D=5, N=2$ supergravity, one obtains [4] 


$$
\begin{aligned}
d s^{2} & =e^{-W}\left(-d t^{2}+d z^{2}\right)+e^{2 W} d \vec{x}^{2}, \\
F_{m n}^{I} & =-\epsilon_{m n p} \partial_{p} H^{I}, \quad e^{W} X^{I}=H^{I}, \\
e^{3 W} & =\frac{1}{6} C_{I J K} H^{I} H^{J} H^{K},
\end{aligned}
$$

with the harmonic functions

$$
H^{I}=h^{I}+\frac{P^{I}}{r}
$$

where $h^{I}$ are constants and $P^{I}$ are magnetic charges.

In the electric case, the near-horizon geometry is given by $A d S_{2} \times S^{3}$ and the black hole entropy, related to the horizon volume $S^{3}$, is given in terms of the extremized electric central charge. For the magnetically charged $D=5$ BPS black string, with near-horizon geometry $A d S_{3} \times S^{2}$, one similarly finds that the extremized value of the BPS tension is related to the volume of the $S^{2}$.

As an example we consider the $S T U=1$ model [1, 18]. This can be obtained by compactification of heterotic string theory on $K_{3} \times S^{1}[\overline{19}]$. The tree-level prepotential of this model is given by

$$
\mathcal{V}=S T U=1
$$

and corresponds to the scalar manifold (2.2) for $n=2$. Taking $S=X^{0}, T=X^{1}$ and $U=X^{2}$, one gets for the matrix $G^{I J}$

$$
G^{I J}=2\left(\begin{array}{ccc}
S^{2} & 0 & 0 \\
0 & T^{2} & 0 \\
0 & 0 & U^{2}
\end{array}\right)
$$

Considering $S$ as the dependent field, i. e. $S=1 /(T U)$, we find

$$
\mathcal{G}_{i j}=\left(\begin{array}{cc}
\frac{1}{T^{2}} & \frac{1}{2 T U} \\
\frac{1}{2 T U} & \frac{1}{U^{2}}
\end{array}\right), \quad \mathcal{G}^{i j}=\frac{4}{3}\left(\begin{array}{cc}
T^{2} & -\frac{T U}{2} \\
-\frac{T U}{2} & U^{2}
\end{array}\right) .
$$

The field equations following from the action (2.4) admit the non-extremal static black hole solution [20]

$$
\begin{aligned}
d s^{2} & =-e^{-4 V} f d t^{2}+e^{2 V}\left(f^{-1} d r^{2}+r^{2} d \Omega_{3}^{2}\right) \\
F_{r t}^{I} & =-H_{I}^{-2} \partial_{r} \tilde{H}_{I} \\
X^{I} & =H_{I}^{-1} e^{2 V}
\end{aligned}
$$

where

$$
d \Omega_{3}^{2}=d \theta^{2}+\sin ^{2} \theta d \phi^{2}+\cos ^{2} \theta d \psi^{2}
$$


denotes the metric on the three sphere $S^{3}$. The $H_{I}$ are harmonic functions given by

$$
H_{I}=1+\frac{Q_{I}}{r^{2}},
$$

and $V$ reads

$$
e^{2 V}=\left(H_{0} H_{1} H_{2}\right)^{1 / 3}
$$

Furthermore we have

$$
f=1-\frac{\mu}{r^{2}}
$$

with the nonextremality parameter $\mu$, and

$$
\tilde{H}_{I}=1+\frac{\tilde{Q}_{I}}{r^{2}}
$$

where the $\tilde{Q}_{I}$ denote the physical electric charges. They are related to the $Q_{I}$ appearing in (3.9) by the equations

$$
\begin{aligned}
Q_{I} & =\frac{\mu}{2} \sinh \beta_{I} \tanh \frac{\beta_{I}}{2}, \\
\tilde{Q}_{I} & =\frac{\mu}{2} \sinh \beta_{I} .
\end{aligned}
$$

The extremal (BPS) limit is reached when $\beta_{I} \rightarrow \infty, \mu \rightarrow 0$, with $\mu \sinh \beta_{I}$ kept fixed. For the ADM mass $M_{A D M}$, the Bekenstein-Hawking entropy $S_{B H}$, and the Hawking temperature $T_{H}$ one obtains

$$
\begin{aligned}
M_{A D M} & =\frac{\pi}{4 G_{5}}\left(\sum_{I} Q_{I}+\frac{3}{2} \mu\right), \\
S_{B H} & =\frac{A_{h o r}}{4 G_{5}}=\frac{\pi^{2}}{2 G_{5}} \prod_{I}\left(\mu+Q_{I}\right)^{1 / 2}, \\
T_{H} & =\frac{\mu}{\pi \prod_{I}\left(\mu+Q_{I}\right)^{1 / 2}} .
\end{aligned}
$$

In the extremal case, also a rotating generalization of (3.7) can be obtained from the general form (3.1). Its metric is given by

$$
d s^{2}=-e^{-4 V}\left(d t+w_{\phi}(r, \theta) d \phi+w_{\psi}(r, \theta) d \psi\right)^{2}+e^{2 V}\left(d r^{2}+r^{2} d \Omega^{2}\right),
$$

where

$$
\begin{aligned}
w_{\phi}(r, \theta) & =-\frac{\alpha \sin ^{2} \theta}{r^{2}}, \\
w_{\psi}(r, \theta) & =\frac{\alpha \cos ^{2} \theta}{r^{2}} .
\end{aligned}
$$


The gauge fields are

$$
A_{t}^{I}=e^{-2 V} X^{I}, \quad A_{\phi}^{I}=e^{-2 V} X^{I} w_{\phi}, \quad A_{\psi}^{I}=e^{-2 V} X^{I} w_{\psi} .
$$

The moduli $X^{I}$ and the functions $V$ and $H_{I}$ are as in (3.7), (3.10) and (3.9) respectively, and the ADM mass is given by (3.14) for $\mu=0$. The Bekenstein-Hawking entropy and the angular momenta read [8]

$$
\begin{aligned}
S_{B H} & =\frac{A_{h o r}}{4 G}=\frac{\pi^{2}}{2 G_{5}} \sqrt{Q_{0} Q_{1} Q_{2}-\alpha^{2}}, \\
J^{\phi} & =-J^{\psi}=\frac{\alpha \pi}{4 G_{5}} .
\end{aligned}
$$

\section{Near-Horizon Limit and Isometry Superalgebra}

In the following two sections, we shall be particularly interested in the near-horizon limit of (3.17). For $r \rightarrow 0$ we can write

$$
e^{2 V}=\frac{Z_{h o r}}{3 r^{2}},
$$

where $Z=Q_{I} X^{I}$ is the central charge, and $Z_{\text {hor }}=3\left(Q_{0} Q_{1} Q_{2}\right)^{1 / 3}$ is its value at the horizon. Introducing the horospherical coordinates $(\tau, \rho)$,

$$
\tau=\frac{t}{2} \sqrt{\frac{3}{Z_{\text {hor }}}}, \quad \rho=2 r^{2} \sqrt{\frac{3}{Z_{\text {hor }}}},
$$

one gets for the near-horizon metric

$$
\begin{aligned}
d s^{2}= & -\rho^{2} d \tau^{2}+\frac{Z_{h o r}}{12 \rho^{2}} d \rho^{2}+\frac{6 \alpha}{Z_{\text {hor }}} \rho d \tau\left(\sin ^{2} \theta d \phi-\cos ^{2} \theta d \psi\right) \\
& +\frac{Z_{\text {hor }}}{3}\left(d \Omega^{2}-\frac{27 \alpha^{2}}{Z_{\text {hor }}^{3}}\left(\sin ^{2} \theta d \phi-\cos ^{2} \theta d \psi\right)^{2}\right) .
\end{aligned}
$$

We observe that, in contrast to the case of vanishing rotation parameter, the spacetime does not split into a product $A d S_{2} \times S^{3}$. Although the $A d S_{2}$ part is the same as without rotation, there are nondiagonal elements, and the three-sphere is distorted. The isometry superalgebra of the near-horizon supergravity configuration was determined in [21], where the fact that the residual isometry supergroup can be determined (modulo bosonic factors) from a knowledge of the Killing spinors [22, 23, 24] has been used. In this way, one obtains that the near-horizon geometry is invariant under the superalgebra $s u(1,1 \mid 2) \oplus u(1)$ in the rotating case, and under $s u(1,1 \mid 2) \oplus s u(2)$ for $\alpha=0$ [21]. Thus, for $\alpha \neq 0$, the bosonic subalgebra is $s u(1,1) \oplus s u(2)_{L} \oplus u(1)_{R}$. In fact, the near-horizon spacetime is a homogeneous manifold of the form $\left[S O(2,1) \times S U(2)_{L} \times U(1)_{R}\right] /[U(1) \times U(1)]$ [21]. The 
conformal algebra $s u(1,1) \cong s o(2,1)$ is generated by the Killing vectors [21]

$$
\begin{aligned}
h & =\partial_{\tau}, \\
d & =\rho \partial_{\rho}-\tau \partial_{\tau}, \\
k & =-\frac{Z_{h o r}}{12 \rho^{2}}\left(1-\frac{27 \alpha^{2}}{Z_{h o r}^{3}} \partial_{\tau}\right)-\tau^{2} \partial_{\tau}+2 \tau \rho \partial_{\rho}-\frac{3 \alpha}{2 Z_{h o r} \rho}\left(\partial_{\phi}-\partial_{\psi}\right),
\end{aligned}
$$

satisfying

$$
[d, h]=h, \quad[d, k]=-k, \quad[h, k]=2 d .
$$

Thus, although the manifold is not a product $A d S_{2} \times S^{3}$, we find the $s o(2,1)$ symmetry inherent to $A d S_{2}$. In the following section, we will see that this symmetry, which is the conformal symmetry in $0+1$ dimensions, occurs also in the action of a particle charged under the vectors moving in the near-horizon regime.

\section{Particle Motion near the Horizon}

We now consider a particle of mass $m$, carrying the charges $q_{I}$ under the abelian vectors, which moves in the background (4.3). Like in [15], we introduce the new coordinate $q$,

$$
\rho=\frac{Z_{\text {hor }}}{3 q^{2}}
$$

We use a Hamiltonian formalism, and define

$$
\mathcal{H}=\frac{1}{2} g^{\mu \nu}\left(\Pi_{\mu}-q_{I} A_{\mu}^{I}\right)\left(\Pi_{\nu}-q_{I} A_{\nu}^{I}\right),
$$

where the $\Pi_{\mu}$ denote generalized momenta. For our configuration, this leads to

$$
\begin{aligned}
\mathcal{H}= & -\frac{9 q^{4}}{2 Z_{\text {hor }}^{2}} \Pi_{\tau}^{2}\left(1-\frac{27 \alpha^{2}}{Z_{\text {hor }}^{3}}\right)+\frac{27 q^{2} \alpha}{Z_{\text {hor }}^{3}} \Pi_{\tau}\left[\Pi_{\phi}-\Pi_{\psi}+\frac{Z_{\text {hor }}^{2}}{9 \alpha} q_{I} X_{\text {hor }}^{I}\right] \\
& -\frac{1}{2}\left(q_{I} X_{\text {hor }}^{I}\right)^{2}+\frac{3 q^{2}}{2 Z_{\text {hor }}} \Pi_{q}^{2}+\frac{3 L^{2}}{2 Z_{\text {hor }}},
\end{aligned}
$$

where

$$
L^{2}=\Pi_{\theta}^{2}+\frac{\Pi_{\phi}^{2}}{\sin ^{2} \theta}+\frac{\Pi_{\psi}^{2}}{\cos ^{2} \theta}
$$

denotes the conserved angular momentum. As the coordinates $\tau, \phi$ and $\psi$ are cyclic, the associated conjugate momenta are constants of motion. If $\mathcal{H}$ solves the mass-shell constraint $2 \mathcal{H}=-m^{2},-\Pi_{\tau}$ is to be identified with the particle Hamiltonian $H$. Setting $\Pi_{q}=p$ and defining $u=p q$, one obtains

$$
H=\frac{p^{2}}{2 F(u)}+\frac{m g}{2 q^{2} F(u)},
$$


with

$$
m g=L^{2}+\frac{Z_{h o r}}{3}\left(m^{2}-\left(q_{I} X_{h o r}^{I}\right)^{2}\right)
$$

and the function $F(u)$ given by

$$
F(u)=\frac{1}{2}\left[\sqrt{C^{2}+\left(1-\frac{27 \alpha^{2}}{Z_{h o r}^{3}}\right)\left(\frac{3}{Z_{h o r}}\left(u^{2}+L^{2}\right)+m^{2}-\left(q_{I} X_{h o r}^{I}\right)^{2}\right)}+C\right] .
$$

In (5.7), the constant $C$ is defined by

$$
C=\frac{9 \alpha}{Z_{h o r}^{2}}\left(\Pi_{\phi}-\Pi_{\psi}\right)+q_{I} X_{h o r}^{I}
$$

One observes that in the limit

$$
Z_{\text {hor }} \rightarrow \infty, \quad m-q_{I} X_{\text {hor }}^{I} \rightarrow 0,
$$

with $Z_{\text {hor }}\left(m-q_{I} X_{h o r}^{I}\right)$ kept fixed, we have $F(u) \rightarrow m$, and (5.5) reduces to the DFF model [25]

$$
H=\frac{p^{2}}{2 m}+\frac{g}{2 q^{2}}
$$

Note that also the general Hamiltonian (5.5) describes a model of conformal mechanics. To see this, we write it in the form

$$
H=\frac{p^{2}}{2 f(u)}
$$

with

$$
f(u)=\frac{u^{2} F(u)}{u^{2}+m g}
$$

The generators of the conformal group are then given by [26]

$$
H=\frac{p^{2}}{2 f}, \quad D=\frac{1}{2} u, \quad K=\frac{1}{2} q^{2} f,
$$

satisfying the Poisson bracket algebra

$$
[D, H]=H, \quad[D, K]=-K, \quad[H, K]=-2 D .
$$




\section{Statistical Entropy from $A d S_{2}$ Central Charge}

In order to determine the central charge of the boundary CFT, we proceed along the lines of [27, 28, 29], and reduce the bosonic part of the $D=5, N=2$ supergravity action to two dimensions. In this section we shall only consider nonrotating black holes carrying electric charge. This means that we can consistently truncate the Chern-Simons term in (2.4), so that the bosonic part of the action in five dimensions reads

$$
I=\frac{1}{16 \pi G_{5}} \int d^{5} x \sqrt{-g}\left[R-\frac{1}{2} G_{I J} F_{\mu \nu}^{I} F^{J \mu \nu}-\mathcal{G}_{i j} \partial_{\mu} \phi^{i} \partial^{\mu} \phi^{j}\right],
$$

where $G_{5}=l^{3}$ denotes Newton's constant. The matrices $G_{I J}$ and $\mathcal{G}_{i j}$ for the $S T U$ model were given in section 3. The reduction ansatz for the metric is

$$
d s^{2}=d s_{(2)}^{2}+l^{2} \Phi^{2} d \Omega^{2},
$$

where $\Phi$ denotes the dilaton and $d \Omega^{2}$ is given by (3.8).

One now assumes that the gauge fields, scalars and dilaton do not depend on the coordinates on the internal $S^{3}$. In this way, one arrives at the two-dimensional effective action

$$
I=\frac{\Omega}{16 \pi} \int d^{2} x \sqrt{-g}\left[\Phi^{3} R+6 \Phi(\nabla \Phi)^{2}+\frac{6 \Phi}{l^{2}}-\Phi^{3} \mathcal{G}_{i j} \partial_{\alpha} \phi^{i} \partial^{\alpha} \phi^{j}-\frac{1}{2} \Phi^{3} G_{I J} F_{\alpha \beta}^{I} F^{J \alpha \beta}\right]
$$

where $\Omega=2 \pi^{2}$ denotes the volume of the unit $S^{3}$, and early greek indices $\alpha, \beta, \ldots$ refer to two-dimensional spacetime. We now wish to integrate out the field strength $F_{\alpha \beta}^{I}$ (which in two dimensions must be a multiple of the volume form $\left.\epsilon_{\alpha \beta}\right)$ from the action. This can be done using the Lagrange multiplier method of [30]. Let us briefly sketch how this works: Instead of looking at the gauge field action

$$
I_{g}=-\frac{\Omega}{16 \pi} \int d^{2} x \sqrt{-g} \frac{1}{2} \Phi^{3} G_{I J} F_{\alpha \beta}^{I} F^{J \alpha \beta},
$$

one looks at the formally extended action

$$
\tilde{I}_{g}=\frac{\Omega}{16 \pi} \int d^{2} x\left[-\frac{1}{2} \sqrt{-g} \Phi^{3} G_{I J} F_{\alpha \beta}^{I} F^{J \alpha \beta}+\lambda_{I}\left(F_{\alpha \beta}^{I}-\partial_{\alpha} A_{\beta}^{I}+\partial_{\beta} A_{\alpha}^{I}\right) \epsilon^{\alpha \beta}\right]
$$

where the definition of $F^{I}$ as a field strength associated with $A^{I}$ is implemented by means of the Lagrange multiplier $\lambda_{I}$. Note that the three variables $F^{I}, A^{I}$ and $\lambda_{I}$ are considered as independent in this setting. Variation with respect to $A^{I}$ yields

$$
\partial_{\beta}\left(\lambda_{I} \epsilon^{\alpha \beta}\right)=0
$$

so that $\Lambda_{I}:=\lambda_{I} / \sqrt{-g}$ are constants. Due to (6.6), the term $\lambda_{I}\left(-\partial_{\alpha} A_{\beta}^{I}+\partial_{\beta} A_{\alpha}^{I}\right) \epsilon^{\alpha \beta}$ in the action (6.5) is a boundary term and can be dropped. We can then integrate out the field strength $F^{I}$, using its equation of motion

$$
-\Phi^{3} G_{I J} F^{J \alpha \beta}+\Lambda_{I} \epsilon^{\alpha \beta}=0 .
$$


This yields

$$
\tilde{I}_{g}=-\frac{\Omega}{16 \pi} \int d^{2} x \sqrt{-g} \frac{G^{I J} \Lambda_{I} \Lambda_{J}}{\Phi^{3}},
$$

so that we are left with the total two-dimensional action

$$
I=\frac{\Omega}{16 \pi} \int d^{2} x \sqrt{-g}\left[\Phi^{3} R+6 \Phi(\nabla \Phi)^{2}+\frac{6 \Phi}{l^{2}}-\Phi^{3} \mathcal{G}_{i j} \partial_{\alpha} \phi^{i} \partial^{\alpha} \phi^{j}-\frac{G^{I J} \Lambda_{I} \Lambda_{J}}{\Phi^{3}}\right] .
$$

The dilaton kinetic term in (6.9) can be eliminated by a conformal rescaling

$$
\bar{g}_{\alpha \beta}=\Phi^{2} g_{\alpha \beta} .
$$

Defining $\bar{\Phi}=\Phi^{3}$, we obtain

$$
I=\frac{\Omega}{16 \pi} \int d^{2} x \sqrt{-\bar{g}}\left[\bar{\Phi} \bar{R}+\frac{6}{l^{2} \bar{\Phi}^{1 / 3}}-\bar{\Phi} \mathcal{G}_{i j} \partial_{\alpha} \phi^{i} \partial^{\alpha} \phi^{j}-\frac{G^{I J} \Lambda_{I} \Lambda_{J}}{\bar{\Phi}^{5 / 3}}\right] .
$$

Let us now consider the nonextremal black hole solution (3.7) of the action (6.1), and expand it near extremality. To this end, we introduce an expansion parameter $\epsilon(\epsilon \rightarrow 0)$, and set

$$
\begin{aligned}
t & =\frac{\tilde{t}}{\epsilon}, \quad r=\sqrt{\epsilon} \tilde{r}, \quad \mu=\mu_{0} \epsilon \\
\bar{\Phi} & =\bar{\Phi}_{0}+\epsilon \varphi, \quad \phi^{i}=\phi_{0}^{i}+\epsilon \tilde{\phi}^{i}
\end{aligned}
$$

where

$$
\bar{\Phi}_{0}=\frac{\left(\tilde{Q}_{0} \tilde{Q}_{1} \tilde{Q}_{2}\right)^{1 / 2}}{l^{3}}, \quad \phi_{0}^{i}=\tilde{Q}_{i}^{-1}\left(\tilde{Q}_{0} \tilde{Q}_{1} \tilde{Q}_{2}\right)^{1 / 3} .
$$

Introducing the new coordinate

$$
x=\frac{\left(\tilde{Q}_{0} \tilde{Q}_{1} \tilde{Q}_{2}\right)^{1 / 6}}{2 l^{2}}\left(\tilde{r}^{2}-\frac{\mu_{0}}{2}\right),
$$

we arrive at

$$
d \bar{s}^{2}=-\left(\lambda^{2} x^{2}-a^{2}\right) d \tilde{t}^{2}+\left(\lambda^{2} x^{2}-a^{2}\right)^{-1} d x^{2}
$$

for the rescaled two-dimensional metric, with $\lambda$ and $a$ given by

$$
\begin{aligned}
\lambda & =\frac{2 l}{\left(\tilde{Q}_{0} \tilde{Q}_{1} \tilde{Q}_{2}\right)^{1 / 3}}, \\
a^{2} & =\frac{\mu_{0}^{2}}{4 l^{2}\left(\tilde{Q}_{0} \tilde{Q}_{1} \tilde{Q}_{2}\right)^{1 / 3}} .
\end{aligned}
$$


Defining a new dilaton $\eta$ by

$$
\eta=\frac{\Omega \epsilon \varphi}{8 \pi},
$$

we obtain for the action at lowest order in the expansion parameter $\epsilon$,

$$
I=\frac{1}{2} \int d^{2} x \sqrt{-\bar{g}} \eta\left[\bar{R}+2 \lambda^{2}\right],
$$

so the leading order is governed by the Jackiw-Teitelboim (JT) model [31. (6.15), together with the linear dilaton

$$
\begin{aligned}
\eta & =\eta_{0} \lambda x, \\
\eta_{0} & =\frac{\Omega \epsilon}{16 \pi l^{2}}\left(\tilde{Q}_{0} \tilde{Q}_{1} \tilde{Q}_{2}\right)^{2 / 3} \sum_{I} \tilde{Q}_{I}^{-1},
\end{aligned}
$$

represents a black hole solution of this model [9], with mass and thermodynamical entropy given by

$$
\begin{aligned}
M_{(2)} & =\frac{1}{2} \eta_{0} a^{2} \lambda, \\
S_{(2)} & =2 \pi \eta_{\text {hor }}=2 \pi \eta_{0} a .
\end{aligned}
$$

This black hole spacetime has constant curvature, i. e. it is locally $A d S_{2}$. Now it is known that the asymptotic symmetries of two-dimensional anti-de Sitter space form a Virasoro algebra [9], similar to the case of $A d S_{3}$, where one has two copies of Virasoro algebras as asymptotic symmetries [32]. When realized canonically in the Hamiltonian formulation of JT gravity, this algebra was shown to exhibit a central charge [9, 33]

$$
c=24 \eta_{0} .
$$

Using this central charge in Cardy's formula, the authors of [9] were able to give a microscopic derivation of the entropy of the two-dimensional black holes (6.15) in the JT model. Our aim is now to perform a similar calculation for the near-extremal five dimensional black hole under consideration, making use of the fact that the dimensionally reduced supergravity action coincides with the JT model at leading order in the nonextremality parameter, and that the relevant two-dimensional metric is given by (6.15) 2 . First of all, we expand the ADM mass $M_{A D M}$ (3.14) and Bekenstein-Hawking entropy $S_{B H}$ (3.15) of the black hole (3.7) in five dimensions for $\mu \rightarrow 0$, yielding

$$
\begin{aligned}
M_{A D M} & =\frac{\pi}{4 l^{3}} \sum_{I} \tilde{Q}_{I}\left(1+\frac{\mu^{2}}{8 \tilde{Q}_{I}^{2}}\right), \\
S_{B H} & =\frac{\Omega}{4 l^{3}}\left(\tilde{Q}_{0} \tilde{Q}_{1} \tilde{Q}_{2}\right)^{1 / 2}\left(1+\frac{\mu}{4} \sum_{I} \tilde{Q}_{I}^{-1}\right),
\end{aligned}
$$

\footnotetext{
${ }^{2}$ Cf. [27 for similar computations in the case of heterotic 4D string black holes.
} 
so the small excitations above extremality have the energy

$$
\Delta M_{A D M}=\frac{\pi \mu^{2}}{32 l^{3}} \sum_{I} \tilde{Q}_{I}^{-1}
$$

and entropy

$$
\Delta S_{B H}=\frac{\Omega \mu}{16 l^{3}}\left(\tilde{Q}_{0} \tilde{Q}_{1} \tilde{Q}_{2}\right)^{1 / 2} \sum_{I} \tilde{Q}_{I}^{-1} .
$$

Comparing this with the two-dimensional results (6.21), one finds $\Delta S_{B H}=S_{(2)}$ and $\Delta M_{A D M}=\epsilon M_{(2)}$. The factor $\epsilon$ appearing in the relation between the two masses stems from the fact that $M_{A D M}$ was computed with respect to the Killing vector $\partial_{t}$, whereas $M_{(2)}$ is related to $\partial_{\tilde{t}}=\epsilon \partial_{t}$. This means that up to these normalizations the five- and two-dimensional energies and entropies match. Expanding also the Hawking temperature (3.16) for small values of the nonextremality parameter $\mu$, one finds for the temperature dependence of $\Delta M_{A D M}$

$$
\Delta M_{A D M}=\frac{\pi^{3} T_{H}^{2}}{32 l^{3}} \tilde{Q}_{0} \tilde{Q}_{1} \tilde{Q}_{2} \sum_{I} \tilde{Q}_{I}^{-1}
$$

so the energy of the excitations above extremality is that of an ideal gas of massless particles in $1+1$ dimensions. This suggests that the microstates should be described by a two-dimensional field theory rather than a quantum mechanical system. Let us now proceed with the computation of the statistical entropy, using the central charge (6.22). The Virasoro generator $L_{0}$ for the black hole (6.15) is given by [9]

$$
L_{0}=\frac{M_{(2)}}{\lambda}=\frac{\Omega \epsilon \mu_{0}^{2}}{128 \pi l^{4}}\left(\tilde{Q}_{0} \tilde{Q}_{1} \tilde{Q}_{2}\right)^{1 / 3} \sum_{I} \tilde{Q}_{I}^{-1} .
$$

Inserting this together with the central charge (6.22) into Cardy's formula, we get for the statistical entropy

$$
S_{\text {stat }}=2 \pi \sqrt{\frac{c L_{0}}{6}}=\frac{\Omega \mu}{8 \sqrt{2} l^{3}}\left(\tilde{Q}_{0} \tilde{Q}_{1} \tilde{Q}_{2}\right)^{1 / 2} \sum_{I} \tilde{Q}_{I}^{-1},
$$

which agrees, up to a factor $\sqrt{2}$, with the thermodynamical entropy $\Delta S_{B H}$ of the small excitations above extremality. The same mismatch by a factor $\sqrt{2}$ has been found in [9], where the authors proposed an explanation of this for the case when the model (6.19) comes from dimensional reduction of three-dimensional AdS gravity. Although in our case $A d S_{2}$ arises as near-horizon geometry of a higher-dimensional black hole with no intermediate $A d S_{3}$ geometry involved, we shall see in the next section that by means of a duality transformation the near-horizon geometry $A d S_{2} \times S^{3}$ of the extremal black hole becomes $A d S_{3} \times S^{2}$. We will then be able to use Strominger's counting of microstates 34 in order to reproduce correctly the Bekenstein-Hawking entropy of the black hole. 


\section{Duality Invariance of the Supergravity Action}

In this section we will show that in presence of a Killing vector field $\partial_{z}$, the supergravity action (6.1) is invariant under a certain generalization of T-dualityp. The key observation is then that the three sphere $S^{3}$ appearing in the black hole geometry can be written as a Hopf fibration, i. e. as an $S^{1}$ bundle over $\mathbb{C} P^{1} \approx S^{2}$. Performing then a duality transformation along the Hopf fibre untwists the $S^{3}$, and transforms the electrically charged black hole into a magnetically charged black string, which has $A d S_{3} \times S^{2}$ as near-horizon limit in the extremal case.

To begin with, we reduce the action (6.1) to four dimensions, using the usual Kaluza-Klein reduction ansatz for the five-dimensional metric,

$$
d s^{2}=e^{k / \sqrt{3}} d s_{4}^{2}+e^{-2 k / \sqrt{3}}\left(d z+\mathcal{A}_{\alpha} d x^{\alpha}\right)^{2},
$$

where $k$ denotes the dilaton, and early greek indices $\alpha, \beta, \ldots$ refer to four-dimensional spacetime. Assuming that the fields appearing in (6.1) are independent of $z$, one arrives at the four-dimensional action

$$
I_{4}=\frac{L}{16 \pi G_{5}} \int d^{4} x \sqrt{-g_{4}}\left[R_{4}-\frac{1}{2}(\nabla k)^{2}-\frac{1}{4} e^{-\sqrt{3} k} \mathcal{F}^{2}-\frac{1}{2} e^{-k / \sqrt{3}} F^{2}-\mathcal{G}_{i j} \partial_{\alpha} \phi^{i} \partial^{\alpha} \phi^{j}\right],
$$

where $L$ denotes the length of the circle parametrized by $z, \mathcal{F}$ is the field strength associated to the Kaluza-Klein vector potential $\mathcal{A}$, and

$$
\mathcal{F}^{2}=\mathcal{F}_{\alpha \beta} \mathcal{F}^{\alpha \beta}, \quad F^{2}=G_{I J} F_{\alpha \beta}^{I} F^{J \alpha \beta} .
$$

We now dualize both $\mathcal{F}$ and $F^{I}$, using again the Lagrange multiplier method of 30. Dropping boundary terms, we arrive at the dualized action

$$
\begin{aligned}
I_{4}= & \frac{L}{16 \pi G_{5}} \int d^{4} x \sqrt{-g_{4}}\left[R_{4}-\frac{1}{2}(\nabla k)^{2}-\frac{1}{4} e^{\sqrt{3} k}\left({ }^{\star} \mathcal{F}\right)^{2}\right. \\
& \left.-\frac{1}{2} e^{k / \sqrt{3}} \frac{1}{4} G^{I J \star} F_{I \alpha \beta}{ }^{\star} F_{J}^{\alpha \beta}-\mathcal{G}_{i j} \partial_{\alpha} \phi^{i} \partial^{\alpha} \phi^{j}\right],
\end{aligned}
$$

where we defined

$$
\begin{aligned}
{ }^{\star} \mathcal{F}_{\alpha \beta} & =\frac{1}{2} e^{-\sqrt{3} k} \epsilon_{\alpha \beta \gamma \delta} \mathcal{F}^{\gamma \delta}, \\
{ }^{\star} F_{I \alpha \beta} & =e^{-k / \sqrt{3}} G_{I J} \epsilon_{\alpha \beta \gamma \delta} F^{J \gamma \delta} .
\end{aligned}
$$

Comparing (7.4) with (7.2), we observe that the gravitational and gauge field parts of the four-dimensional action, as well as the dilaton kinetic energy, are invariant under the $\mathbb{Z}_{4}$ transformation

$$
k \rightarrow-k, \quad \mathcal{F}_{\alpha \beta} \rightarrow{ }^{\star} \mathcal{F}_{\alpha \beta}, \quad F_{\alpha \beta}^{I} \rightarrow{ }^{\star} F_{I \alpha \beta}, \quad G_{I J} \rightarrow \frac{1}{4} G^{I J} .
$$

\footnotetext{
${ }^{3}$ By considering (6.1) we assumed that the Chern-Simons term does not contribute. One can easily generalize the discussion below to nonvanishing CS term. This results in a $\theta$ term in four dimensions, which does not spoil the considered duality invariance.
} 
The $\mathbb{Z}_{4}$ is actually a subgroup of the usual symplectic $S p(2 m+2, \mathbb{R})$ duality group [35, 36] of $D=4, N=2$ supergravity (coupled to $m$ vector multiplets) generated by

$$
S=\left(\begin{array}{cc}
0 & 1 \\
-1 & 0
\end{array}\right) \text {. }
$$

Note that the transformation $G_{I J} \rightarrow G^{I J} / 4$ means that

$$
\begin{aligned}
& X^{I} \rightarrow 3 X_{I}=\frac{1}{2} C_{I J K} X^{J} X^{K}, \\
& X_{I} \rightarrow \frac{1}{3} X^{I},
\end{aligned}
$$

so essentially the special coordinates go over into their duals. The fact that this dualization implies $G_{I J} \rightarrow G^{I J} / 4$ can be shown using the expression

$$
G_{I J}=\frac{9}{2} X_{I} X_{J}-\frac{1}{2} C_{I J K} X^{K},
$$

as well as the "adjoint identity"

$$
C_{I J K} C_{J^{\prime}(L M} C_{P Q) K^{\prime}} \delta^{J J^{\prime}} \delta^{K K^{\prime}}=\frac{4}{3} \delta_{I(L} C_{M P Q)}
$$

of the associated Jordan algebra [17]. It can also be seen that this duality transformation is consistent with the relations (2.6). Furthermore, making use of the equation

$$
\mathcal{G}_{i j} \partial_{\alpha} \phi^{i} \partial^{\alpha} \phi^{j}=G_{I J} \partial_{\alpha} X^{I} \partial^{\alpha} X^{J},
$$

one checks that (7.9) does not change the kinetic term of the scalar fields, so (7.7), (7.9) represent in fact a duality invariance of the four-dimensional action (7.2). In the special case of the $S T U=1$ model, (7.9) implies that the moduli $\phi^{i}$ go over into their inverse,

$$
\phi^{i} \rightarrow \frac{1}{\phi^{i}}
$$

We now wish to apply the duality (77.7), (77.9) to the black hole solution (3.7). To this end, we consider the $S^{3}$ as an $S^{1}$ bundle over $S^{2}$, and write for its metric

$$
d \Omega^{2}=\frac{1}{4}\left[d \vartheta^{2}+\sin ^{2} \vartheta d \varphi^{2}+(d \zeta+\cos \vartheta d \varphi)^{2}\right],
$$

where $\zeta(0 \leq \zeta \leq 4 \pi)$ parametrizes the $S^{1}$ fibre. Introducing the coordinate $z=\lambda \zeta$, where $\lambda$ denotes an arbitrary length scale, one can write the $5 \mathrm{~d}$ metric in the KK form (7.1), where

$$
\begin{aligned}
d s_{4}^{2} & =\frac{r e^{V}}{2 \lambda}\left[-e^{-4 V} f d t^{2}+e^{2 V} f^{-1} d r^{2}+e^{2 V} \frac{r^{2}}{4}\left(d \vartheta^{2}+\sin ^{2} \vartheta d \varphi^{2}\right)\right], \\
e^{-k / \sqrt{3}} & =\frac{r e^{V}}{2 \lambda} \\
\mathcal{A} & =\lambda \cos \vartheta d \varphi .
\end{aligned}
$$


(Note that $\mathcal{F}=d \mathcal{A}$ is essentially the Kähler form on $S^{2}$ ). We now dualize in $4 \mathrm{~d}$ according to (7.7), and then relift the solution to five dimensions. This yields the configuration

$$
\begin{aligned}
d s^{2} & =e^{-2 V}\left[\frac{\mu}{4 \lambda^{2}} d t^{2}+2 d z d t+\frac{4 \lambda^{2}}{r^{2}} d z^{2}\right]+\frac{r^{2}}{4 \lambda^{2}} e^{4 V}\left[f^{-1} d r^{2}+\frac{r^{2}}{4} d \Omega_{2}^{2}\right], \\
F_{\vartheta \varphi}^{I} & =\frac{\tilde{Q}_{I}}{4 \lambda} \sin \vartheta \\
X^{I} & =H_{I} e^{-2 V} .
\end{aligned}
$$

One effect of the duality transformation is thus the untwisting of the Hopf fibration Although the metric in (7.16) contains nondiagonal elements proportional to $d z d t$, there is no rotation present. To see this, one observes that the nondiagonal elements come from the vector potential $\mathcal{A}$ in four dimensions, which gives rise to the field strength $\mathcal{F}$. The equations of motion for $\mathcal{F}$ following from the action $(7.2)$ read

$$
\nabla_{\alpha}\left(e^{-k \sqrt{3}} \mathcal{F}^{\alpha \beta}\right)=0
$$

so there exists an associated conserved charge

$$
J=\int_{S_{\infty}^{2}} d^{2} S_{\alpha \beta} e^{-k \sqrt{3}} \mathcal{F}^{\alpha \beta} .
$$

For the solution (7.16) under consideration, however, one easily verifies that $J$ (which, up to a normalization factor, represents the angular momentum) vanishes.

One can further simplify (7.16) by an $S L(2, \mathbb{R})$ transformation

$$
\left(\begin{array}{l}
t^{\prime} \\
z^{\prime}
\end{array}\right)=\left(\begin{array}{cc}
0 & -\frac{2 \lambda}{\sqrt{\mu}} \\
\frac{\sqrt{\mu}}{2 \lambda} & \frac{2 \lambda}{\sqrt{\mu}}
\end{array}\right)\left(\begin{array}{l}
t \\
z
\end{array}\right) .
$$

Introducing also the new radial coordinate $\rho=r^{2} /(4 \lambda)$, we then get for the metric

$$
d s^{2}=e^{-2 V}\left(-f d t^{\prime 2}+d z^{2}\right)+e^{4 V}\left(f^{-1} d \rho^{2}+\rho^{2} d \Omega_{2}^{2}\right) .
$$

$(7.20)$, together with the gauge and scalar fields given in (7.16), represents a nonextremal generalization of the supersymmetric magnetic black string found in [⿴囗十 . The duality (7.7) thus maps electrically charged black holes onto magnetically charged black strings. Now a short comment on the $S L(2, \mathbb{R})$ transformation $(7.19)$ is in order. The orbits of the Killing vector

$$
\partial_{z^{\prime}}=\frac{2 \lambda}{\sqrt{\mu}} \partial_{t}
$$

\footnotetext{
${ }^{4}$ The fact that Hopf bundles can be untwisted by T-dualities was observed in [37, 38]. The idea of untwisting and twisting fibres to relate strings and black holes, and thus to gain new insights into black hole microscopics, was also explored in [39].
} 
are non-compact since the time coordinate is non-compact. This means that globally the spacetimes in (7.16) and (7.20) are not equivalent. To make the transformation (7.19) a symmetry, we have to compactify the orbits of $\partial_{z^{\prime}}$. We shall see below however, that the temperature and entropy of one black string can be deduced from the other, which indicates that the two solutions (7.16) and (7.20) are in the same universality class [40]. The Bekenstein-Hawking entropy of the black string (7.20) results to coincide precisely with that of the dual black hole given by (3.15), if we assign to $z^{\prime}$ the period $\Delta z 2 \lambda / \sqrt{\mu}$, where $\Delta z=4 \pi \lambda$ denotes the period of $z$. The Hawking temperature can be computed by requiring the absence of conical singularities in the Euclidean metric, yielding

$$
T_{H}=\frac{2 \lambda \sqrt{\mu}}{\pi \prod_{I}\left(\mu+Q_{I}\right)^{1 / 2}},
$$

i. e. $2 \lambda / \sqrt{\mu}$ times the black hole temperature (3.16). The factor $2 \lambda / \sqrt{\mu}$ stems from the rescaling of the time coordinate contained in (7.19). Thus, up to this normalization, the temperature and entropy of the black string $(7.20)$ coincide with that of the dual black hole (3.7), i. e. they are duality invariant.

\section{Microstate Counting from $A d S_{3}$ Gravity}

We now want to use the near-horizon geometry of the dual solution $(7.20)$ to count the microstates giving rise to the Bekenstein-Hawking entropy. In [4] it was shown that in the extremal case, the geometry becomes $A d S_{3} \times S^{2}$ near the event horizon. The idea is now to use the central charge of $A d S_{3}$ gravity 32 in Cardy's formula, in order to compute the statistical entropy, like it was done by Strominger [34] for the BTZ black holef. As only the $A d S_{3}$ part is relevant, we would like to reduce the supergravity action from five to three dimensions. To this end, we first Hodge-dualize the magnetic two-form field strength in (7.16). This yields for the action (6.1)

$$
I=\frac{1}{16 \pi G_{5}} \int d^{5} x \sqrt{-g}\left[R-\frac{1}{2} G^{I J} H_{I \mu \nu \rho} H_{J}^{\mu \nu \rho}-\mathcal{G}_{i j} \partial_{\mu} \phi^{i} \partial^{\mu} \phi^{j}\right],
$$

where

$$
H_{I \mu \nu \rho}=-\frac{1}{2 \sqrt{3}} G_{I J} \epsilon_{\mu \nu \rho \lambda \sigma} F^{J \lambda \sigma} .
$$

Note that for the solution under consideration, the $H_{I}$ do not depend on the coordinates of the internal $S^{2}$. Furthermore, in 3d the three-forms $H_{I}$ are proportional to the volume form and can be integrated out. For the metric, we use the reduction ansatz

$$
d s^{2}=d s_{3}^{2}+l^{2} \Phi^{2} d \Omega_{2}^{2}
$$

\footnotetext{
${ }^{5}$ Cf. also 41], where similar computations for black strings in six dimensions with $B T Z \times S^{3}$ nearhorizon geometry were performed.
} 
where $G_{5}=l^{3}$ as before, and $d \Omega_{2}^{2}$ denotes the standard metric on the unit $S^{2}$. This gives the reduced action

$$
I=\frac{1}{4 l} \int d^{3} x \sqrt{-g_{3}} \Phi^{2}\left[R_{3}+\frac{2}{l^{2} \Phi^{2}}+\frac{2}{\Phi^{2}}(\nabla \Phi)^{2}-\frac{1}{2} G^{I J} H_{I \alpha \beta \gamma} H_{J}^{\alpha \beta \gamma}-\mathcal{G}_{i j} \partial_{\alpha} \phi^{i} \partial^{\alpha} \phi^{j}\right],
$$

where early greek indices $\alpha, \beta, \ldots$ refer to three-dimensional spacetime. Using the procedure described in section 6, the three-forms $H_{I}$ can be integrated out. In this way, one finally obtains

$$
I=\frac{1}{4 l} \int d^{3} x \sqrt{-g_{3}} \Phi^{2}\left[R_{3}+\frac{2}{l^{2} \Phi^{2}}+\frac{2}{\Phi^{2}}(\nabla \Phi)^{2}-\frac{G_{I J} P^{I} P^{J}}{\Phi^{4} l^{4}}-\mathcal{G}_{i j} \partial_{\alpha} \phi^{i} \partial^{\alpha} \phi^{j}\right],
$$

where we introduced the magnetic charges

$$
P^{I}=\frac{\tilde{Q}_{I}}{4 \lambda}
$$

of the black string $(\overline{7.16})$.

We find it convenient to conformally rescale the metric,

$$
\bar{g}_{\alpha \beta}=\Phi g_{\alpha \beta},
$$

yielding

$$
I=\frac{1}{4 l} \int d^{3} x \sqrt{-\bar{g}_{3}} \Phi^{\frac{3}{2}}\left[\bar{R}_{3}+\frac{2}{l^{2} \Phi^{3}}-\frac{3}{2 \Phi^{2}}(\nabla \Phi)^{2}-\frac{G_{I J} P^{I} P^{J}}{\Phi^{5} l^{4}}-\mathcal{G}_{i j} \partial_{\alpha} \phi^{i} \partial^{\alpha} \phi^{j}\right]
$$

for the action. The conformally rescaled $3 \mathrm{~d}$ metric reads

$$
d \bar{s}_{3}^{2}=\frac{\rho}{l}\left(-f d t^{\prime 2}+d z^{\prime 2}\right)+e^{6 V} \frac{\rho d \rho^{2}}{l f} .
$$

The idea is now to expand this metric near the horizon and near extremality. This can be done by setting

$$
t^{\prime}=\frac{t^{\prime \prime}}{\sqrt{\epsilon}}(2 \lambda)^{4} \sqrt{\frac{l}{\mu_{0} \lambda \tilde{Q}_{0} \tilde{Q}_{1} \tilde{Q}_{2}}}, \quad z^{\prime}=\frac{z^{\prime \prime}}{\sqrt{\epsilon}} \frac{(2 \lambda)^{2}}{\sqrt{\mu_{0}}}, \quad \rho=\epsilon \tilde{r}^{2} \frac{\mu_{0} l}{(2 \lambda)^{4}}, \quad \mu=\mu_{0} \epsilon,
$$

and taking the limit $\epsilon \rightarrow 0$. This leads to the metric

$$
d \bar{s}_{3}^{2}=-\frac{\tilde{r}^{2}-\tilde{r}_{+}^{2}}{l_{e f f}^{2}} d t^{\prime \prime 2}+\tilde{r}^{2} d z^{\prime \prime 2}+\frac{l_{e f f}^{2} d \tilde{r}^{2}}{\tilde{r}^{2}-\tilde{r}_{+}^{2}},
$$

where we introduced

$$
\begin{aligned}
\tilde{r}_{+}^{2} & =\frac{4 \lambda^{3}}{l} \\
l_{e f f}^{2} & =\frac{\tilde{Q}_{0} \tilde{Q}_{1} \tilde{Q}_{2}}{16 l \lambda^{3}} .
\end{aligned}
$$


We recognize (8.11) as the BTZ black hole [42, with event horizon at $\tilde{r}=\tilde{r}_{+}$. One easily verifies that the period of the coordinate $z^{\prime \prime}$ is $2 \pi . \Lambda_{e f f}=-1 / l_{e f f}^{2}$ is the effective cosmological constant. The effective $3 \mathrm{~d}$ Newton constant can be read off from the action (8.8), yielding

$$
\frac{1}{16 \pi G_{\text {eff }}}=\frac{1}{4 l} \Phi_{\text {hor }}^{3 / 2}
$$

where the subscript indicates that the dilaton $\Phi$ is to be evaluated at the horizon. In this way, we get

$$
\frac{1}{G_{\text {eff }}}=\frac{4 \pi}{l^{5 / 2}} e^{3 V_{\text {hor }}} \rho_{\text {hor }}^{3 / 2} .
$$

The Bekenstein-Hawking entropy of the BTZ black hole (8.11) is given by

$$
S_{(3)}=\frac{A_{\text {hor }}}{4 G_{\text {eff }}}=\frac{\pi^{2}}{2 l^{3}} \prod_{I}\left(\mu+Q_{I}\right)^{1 / 2},
$$

which, as it should be, equals the entropy (3.15) of the five-dimensional black hole we started with. The BTZ black hole mass $M_{(3)}$ can be computed using the formula

$$
\tilde{r}_{+}^{2}=8 G_{e f f} M_{(3)} l_{e f f}^{2}
$$

which yields

$$
M_{(3)}=\frac{\lambda^{3}}{2 l G_{e f f} l_{e f f}^{2}} .
$$

We can now apply Strominger's counting of microstates 34 to reproduce the BekensteinHawking entropy. To this end, one first observes that the central charge appearing in the asymptotic symmetry algebra of $A d S_{3}$ [32] in our case reads

$$
c=\frac{3 l_{\text {eff }}}{2 G_{\text {eff }}} .
$$

Furthermore, we have the relations

$$
\begin{aligned}
M_{(3)} & =\frac{1}{l_{e f f}}\left(L_{0}+\bar{L}_{0}\right), \\
J & =L_{0}-\bar{L}_{0}
\end{aligned}
$$

for the mass and angular momentum. For (8.11) one has $J=0$, so $L_{0}=\bar{L}_{0}=\frac{1}{2} l_{\text {eff }} M_{(3)}$. Plugging this, together with the central charge (8.18), into Cardy's formula

$$
S_{\text {stat }}=2 \pi \sqrt{\frac{c L_{0}}{6}}+2 \pi \sqrt{\frac{c \bar{L}_{0}}{6}}
$$

yields the statistical entropy

$$
S_{\text {stat }}=\frac{\pi \lambda^{3 / 2}}{l^{1 / 2} G_{e f f}}=\frac{\pi^{2}}{2 l^{3}} \prod_{I}\left(\mu+Q_{I}\right)^{1 / 2},
$$

which coincides precisely with the thermodynamical entropy (3.15) of the 5d black hole (3.7). 


\section{$9 \quad$ Final Remarks}

The conclusions we have drawn are valid for general black holes of $D=5, N=2$ supergravities. In particular they apply also to the case of theories obtained from compactifications on Calabi-Yau spaces. In different contexts there has been a discussion of dualities [43, 44, 45] which connect various black hole solutions. We have exhibited an explicit duality transformation which is an invariance of the action: it turns the $A d S_{2} \times S^{3}$ near horizon geometry into $A d S_{3} \times S^{2}$.

Our calculation shows that the correct statistical entropy is given by the counting of microstates from $A d S_{3}$, where both $L_{0}$ and $\bar{L}_{0}$ are different from zero. Using instead the central charge of the $A d S_{2}$ Virasoro algebra, with only right-movers, gives a factor $\sqrt{2}$ mismatch between statistical and thermodynamical entropy. Within the $A d S_{2}$ approach

we were able (up to the mentioned factor $\sqrt{2}$ ) to capture only the entropy of the small excitations above extremality, not that of the ground state itself. The reason for this was the fact that in two dimensions the Einstein-Hilbert term is a topological invariant, and does not contribute to the central charge computed in [9]. In the extremal limit $\epsilon \rightarrow 0$ the $A d S_{2}$ central charge (6.22) vanishes, whereas the central charge (8.18) for $A d S_{3}$ is given by

$$
c=\frac{3 \pi}{16 l^{3} \lambda^{3}} \tilde{Q}_{0} \tilde{Q}_{1} \tilde{Q}_{2}
$$

This is in agreement with Strominger's observation [46] that the $A d S_{2}$ Virasoro algebra is related to the right-moving $A d S_{3}$ Virasoro algebra by a topological twist which shifts the central charge to zero.

It might be that the degeneracy of the ground state itself is effectively captured by a model of conformal quantum mechanics [16]. However, our results support the point of view that the excitations above extremality are described by a two-dimensional conformal field theory [47, 11]. 


\section{References}

[1] A. Chou, R. Kallosh, J. Rahmfeld, S. -J. Rey, M. Shmakova, and W. K. Wong, Critical Points and Phase Transitions in 5D Compactifications of M-Theory, Nucl. Phys. B508 (1997) 147.

[2] I. Gaida, S. Mahapatra, T. Mohaupt, and W. A. Sabra, Black Holes and Flop Transitions in M-Theory on Calabi-Yau Threefolds, Class. Quant. Grav. 16 (1999) 419.

[3] B. de Wit and A. Van Proeyen, Broken Sigma-Model Isometries in Very Special Geometry, Phys. Lett. B293 (1992) 94.

[4] A. H. Chamseddine and W. A. Sabra, Calabi-Yau Black Holes and Enhancement of Supersymmetry in Five Dimensions, Phys. Lett. B460 (1999) 63.

[5] M. Cvetič and D. Youm, General Rotating Five Dimensional Black Holes of Toroidally Compactified Heterotic String, Nucl. Phys. B476 (1996) 118;

Entropy of Non-Extreme Charged Rotating Black Holes in String Theory, Phys. Rev. D54 (1996) 2612.

[6] J. C. Breckenridge, R. C. Myers, A. W. Peet, and C. Vafa, D-Branes and Spinning Black Holes, Phys. Lett. B391 (1997) 93.

[7] R. Kallosh, A. Rajaraman, and W. K. Wong, Supersymmetric Rotating Black Holes and Attractors, Phys. Rev. D55 (1997) 3246.

[8] A. H. Chamseddine and W. A. Sabra, Metrics Admitting Killing Spinors in Five Dimensions, Phys. Lett. B426 (1998) 36.

[9] M. Cadoni and S. Mignemi, Entropy of 2D Black Holes from Counting Microstates, Phys. Rev. D59 (1999) 081501;

Asymptotic Symmetries of $A d S_{2}$ and Conformal Group in $D=1$, Nucl. Phys. B557 (1999) 165.

[10] J. M. Maldacena, J. Michelson, and A. Strominger, Anti-de Sitter Fragmentation, JHEP 9902 (1999) 011.

[11] J. M. Maldacena and A. Strominger, Universal Low-Energy Dynamics for Rotating Black Holes, Phys. Rev. D56 (1997) 4975.

[12] J. M. Maldacena, The Large $N$ Limit of Superconformal Field Theories and Supergravity, Adv. Theor. Math. Phys. 2 (1998) 231.

[13] C. Vafa, Black Holes and Calabi-Yau Threefolds, Adv. Theor. Math. Phys. 2 (1998) 207.

[14] A. Strominger and C. Vafa, Microscopic Origin of the Bekenstein-Hawking Entropy, Phys. Lett. B379 (1996) 99. 
[15] P. Claus, M. Derix, R. Kallosh, J. Kumar, P. K. Townsend, and A. Van Proeyen, Black Holes and Superconformal Mechanics, Phys. Rev. Lett. 81 (1998) 4553.

[16] G. W. Gibbons and P. K. Townsend, Black Holes and Calogero Models, Phys. Lett. B454 (1999) 187.

[17] M. Günaydin, G. Sierra, and P. K. Townsend, The Geometry of $N=2$ MaxwellEinstein Supergravity and Jordan Algebras, Nucl. Phys. B242 (1984) 244.

[18] W. A. Sabra, General BPS Black Holes in Five Dimensions, Mod. Phys. Lett. A13 (1998) 239.

[19] I. Antoniadis, S. Ferrara, and T. R. Taylor, $N=2$ Heterotic Superstring and its Dual Theory in Five Dimensions, Nucl. Phys. B460 (1996) 489.

[20] K. Behrndt, M. Cvetič, and W. A. Sabra, Non-Extreme Black Holes of Five Dimensional $N=2$ AdS Supergravity, Nucl. Phys. B553 (1999) 317.

[21] J. P. Gauntlett, R. C. Myers, and P. K. Townsend, Black Holes of D=5 Supergravity, Class. Quant. Grav. 16 (1999) 1.

[22] J. P. Gauntlett, R. C. Myers, and P. K. Townsend, Supersymmetry of Rotating Branes, Phys. Rev. D59 (1999) 025001.

[23] P. K. Townsend, Killing Spinors, Supersymmetries and Rotating Intersecting Branes, hep-th/9901102, to appear in Proceedings of the 22nd Johns Hopkins Workshop, Gothenburg, 1998.

[24] J. M. Figueroa-O'Farrill, On the Supersymmetries of Anti-de Sitter Vacua, Class. Quant. Grav. 16 (1999) 2043.

[25] V. De Alfaro, S. Fubini, and G. Furlan, Conformal Invariance in Quantum Mechanics, Nuovo Cimento 34A (1976) 569.

[26] J. Kumar, Conformal Mechanics and the Virasoro Algebra, JHEP 9904 (1999) 006.

[27] M. Cadoni, Dimensional Reduction of $4 D$ Heterotic String Black Holes, Phys. Rev. D60 (1999) 084016.

[28] S. Cacciatori, D. Klemm, and D. Zanon, $w_{\infty}$ Algebras, Conformal Mechanics, and Black Holes, hep-th/9910065.

[29] J. Navarro-Salas and P. Navarro, AdS $S_{2} / C F T_{1}$ Correspondence and Near-Extremal Black Hole Entropy, hep-th/9910076.

[30] N. Kaloper, Topological Mass Generation in Three Dimensional String Theory, Phys. Lett. B320 (1994) 16. 
[31] C. Teitelboim, in Quantum Theory of Gravity, edited by S. M. Christensen (Hilger, Bristol); R. Jackiw, ibid.

[32] J. D. Brown and M. Henneaux, Central Charges in the Canonical Realization of Asymptotic Symmetries: An Example from Three-Dimensional Gravity, Commun. Math. Phys. 104 (1986) 207.

[33] M. Cadoni and S. Mignemi, Symmetry Breaking, Central Charges and the $A d S_{2} / C F T_{1}$ Correspondence, hep-th/0002256.

[34] A. Strominger, Black Hole Entropy from Near-Horizon Microstates, JHEP 9802 (1998) 009.

[35] M. K. Gaillard and B. Zumino, Duality Rotations for Interacting Fields, Nucl. Phys. B193 (1981) 221.

[36] B. Craps, F. Roose, W. Troost, and A. Van Proeyen, What is Special Kähler Geometry?, Nucl. Phys. B503 (1997) 565.

[37] M. J. Duff, H. Lü, and C. N. Pope, $A d S_{5} \times S^{5}$ Untwisted, Nucl. Phys. B532 (1998) 181

$A d S_{3} \times S^{3}$ (Un)twisted and Squashed, and an $O(2,2 ; \mathbb{Z})$ Multiplet of Dyonic Strings, Nucl. Phys. B544 (1999) 145.

[38] E. Halyo, Supergravity on Ad $S_{5 / 4} \times$ Hopf Fibrations and Conformal Field Theories, hep-th/9803193.

[39] M. Cvetič, H. Lü, and C. N. Pope, Decoupling Limit, Lens Spaces and Taub-NUT: $D=4$ Black Hole Microscopics from D = 5 Black Holes, Nucl. Phys. B549 (1999) 194.

[40] K. Skenderis, Black Holes and Branes in String Theory, Erice lecture notes, hepth/9901050.

[41] M. Cvetič and F. Larsen, Near Horizon Geometry of Rotating Black Holes in Five Dimensions, Nucl. Phys. B531 (1998) 239.

[42] M. Bañados, C. Teitelboim, and J. Zanelli, Black Hole in Three-Dimensional Spacetime, Phys. Rev. Lett. 69 (1992) 1849.

[43] K. Sfetsos and K. Skenderis, Microscopic Derivation of the Bekenstein-Hawking Entropy Formula for Non-Extremal Black Holes, Nucl. Phys. B517 (1998) 179.

[44] R. Argurio, F. Englert, and L. Houart, Statistical Entropy of the Four-Dimensional Schwarzschild Black Hole, Phys. Lett. B426 (1998) 275.

[45] B. Julia, Superdualities: Below and Beyond U-duality, hep-th/0002035. 
[46] A. Strominger, AdS $S_{2}$ Quantum Gravity and String Theory, JHEP 9901 (1999) 007.

[47] G. T. Horowitz and A. Strominger, Counting States of Near-Extremal Black Holes, Phys. Rev. Lett. 77 (1996) 2368;

G. T. Horowitz, D. A. Lowe, and J. M. Maldacena, Statistical Entropy of Nonextremal Four-Dimensional Black Holes and U-Duality, Phys. Rev. Lett. 77 (1996) 430. 